Document downloaded from:

http://hdl.handle.net/10251/92363

This paper must be cited as:

Espinosa Soria, A.; Rodríguez Fortuño, FJ.; Griol Barres, A.; Martínez Abietar, AJ. (2017). On-Chip Optimal Stokes Nanopolarimetry Based on Spin-Orbit Interaction of Light. Nano Letters. 17(5):3139-3144. doi:10.1021/acs.nanolett.7b00564

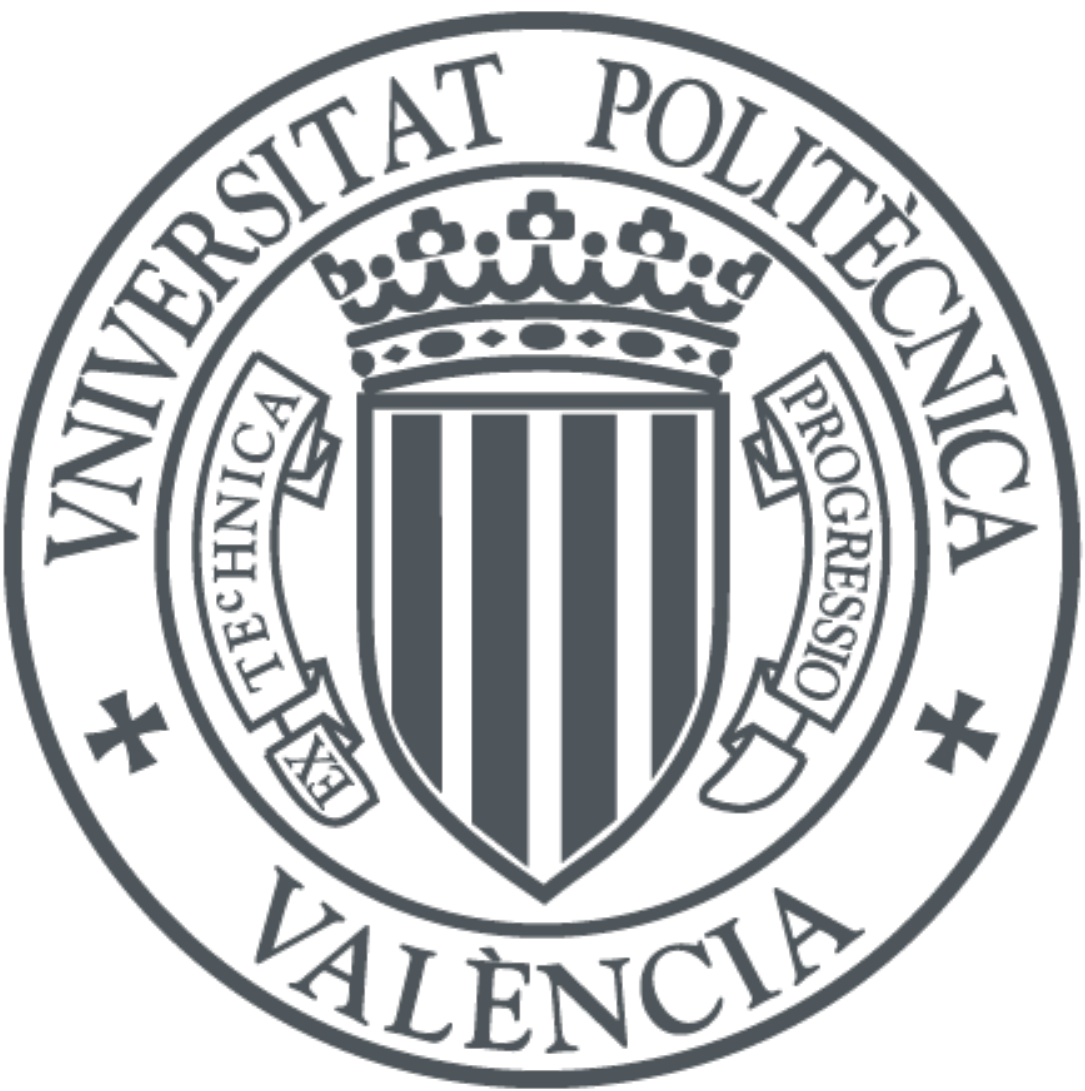

The final publication is available at

http://doi.org/10.1021/acs.nanolett.7b00564

Copyright American Chemical Society

Additional Information 


\title{
On-chip optimal Stokes nanopolarimetry based on spin-orbit interaction of light
}

\author{
Alba Espinosa-Soria ${ }^{1}$, Francisco J.Rodríguez-Fortuño ${ }^{2}$, Amadeu Griol ${ }^{1}$ and Alejandro Martínez ${ }^{1 *}$ \\ ${ }^{1}$ Nanophotonics Technology Center, Universitat Politècnica de València, Valencia, Spain \\ ${ }^{2}$ Department of Physics, King's College London, Strand, London WC2R 2LS, United Kingdom \\ * email: amartinez@ntc.upv.es
}

\begin{abstract}
Full measurement of the polarization of light at the nanoscale is expected to be crucial in many scientific and technological disciplines. Ideally, such measurements will require miniaturized Stokes polarimeters able to determine polarization nondestructively, locally and in real time. For maximum robustness in measurement, the polarimeters should also operate optimally. Recent approaches making use of plasmonic nanostructures or metasurfaces are not able to fulfil all these requirements simultaneously. Here, we propose and demonstrate a method for subwavelengthfootprint Stokes nanopolarimetry based on spin-orbit interaction of light. The method, which basically consists on a subwavelength scatterer coupled to a (set of) multimode waveguide(s), can fully determine the state of polarization satisfying all the previous features. Remarkably, the nanopolarimetry technique can operate optimally (we design a nanopolarimeter whose polarization basis spans $99.7 \%$ of the maximum tetrahedron volume inside the Poincaré sphere) over a broad bandwidth. Although here experimentally demonstrated on a silicon chip at telecom wavelengths, spin-orbit-interaction-based nanopolarimetry is a universal concept to be applied in any wavelength regime or technological platform.
\end{abstract}

Keywords: polarimetry; spin-orbit interaction; nanoantenna; silicon waveguide; nanophotonics. 
While apparently unrelated, the propagation direction and the polarization of a light beam may be strongly connected via spin-orbit interaction (SOI) of light. ${ }^{1-10}$ Indeed, SOI is ultimately responsible for the unidirectional propagation of guided waves controlled by the spin of an excitation source. ${ }^{4,5}$ Spin-controlled directional guiding of light has been demonstrated in a wide variety of guiding systems, including metal plates ${ }^{4}$, optical fibers ${ }^{6}$ and integrated waveguides. ${ }^{7,8}$ In these systems, SOI takes place when placing a certain emitting (i.e., a quantum dot) or scattering (i.e. a subwavelength scatterer) element that acts as a spin-controlled point-like source in a region where the waveguide mode has a non-negligible local transverse spin. ${ }^{9}$ Unlike plane waves (exclusively carrying longitudinal spin), evanescent waves present in guided fields display a strong transverse spin that is locked to their momentum, so spin-controlled directional guiding can be ultimately considered as a manifestation of the quantum spin Hall effect (QSHE) of light. ${ }^{1,10-11}$

SOI in scatterer-waveguide systems is not limited to the separation of circular polarizations, as considered in previous experiments $s^{4-8}$. Indeed, careful engineering of the scatterer enables sorting linearly polarized photons as long as the incident radiation induces a spinning field in it. ${ }^{12}$ In a broad sense, $\mathrm{SOI}$ in systems showing spin-momentum locking allows mapping the polarization of a light beam into different amplitudes of guided waves propagating along different optical paths or modes. This should enable the recovery of the state of polarization (SoP) of the incoming wave, which is fully described by the Stokes vector $\mathbf{S}=\left(S_{0}, S_{1}, S_{2}, S_{3}\right)^{\mathrm{T}}$, as long as a sufficient number of optical paths are measured. Since the scatterer can be much smaller than the wavelength (ultimately a single atom $^{13}$ ), SoP would be measured at a local level, thus allowing for detecting the SoP in nanoscale systems as well as in macro-scale wave fronts with spatially-variant polarization. ${ }^{14,15}$ Therefore, SOI may become a key tool in polarimetry at the nanoscale by enabling extreme shrinking -with all its inherent advantages ${ }^{16}$ - of polarimeters (nanopolarimetry). Here, we introduce a method for Stokes nanopolarimetry making use of the SOI of light and experimentally demonstrate it at telecom wavelengths on a silicon chip.

SOI Stokes nanopolarimetry relies on placing a subwavelength scatterer in the close proximity of one or more multimode waveguides, being the system illuminated from the top by a transverse light beam (see Fig. 1a). In general, each waveguide port may support several guided modes, forming a total of $N$ port-mode combinations, each of them having different SOI behavior associated to the $\mathrm{QSHE}^{17}$. Crucially, SOI allows the power excited onto each port-mode combination to be different according to the incident SoP. We define the effective area of the scatterer $A_{\mathrm{eff}}^{k}(\mathbf{E})$ as the power scattered into the $k$-th port-mode combination, divided by the intensity of the incident illumination $I_{\text {inc }}$ with polarization $\mathbf{E}$. Measuring the power on each port-mode combination, we collect them in a real vector $\mathbf{P}$. By injecting a proper set of input polarizations (calibration process) we can easily form 
the polarimetric matrix $\mathbf{W}$ so that $\mathbf{P}=\mathbf{W} \cdot \mathbf{S}{ }^{18}$. Then, the full SoP of any input polarization could be retrieved as $\mathbf{S}=\overline{\mathbf{W}}^{-1} \cdot \mathbf{P}$ as long as $\mathbf{N} \geq 4$, so our device performs as a Stokes nanopolarimeter (see the full discussion in the Supporting Information, Section S.2).

In its simplest form, the scatterer is coupled to a single waveguide supporting two propagation directions with (at least) two guided modes each. For instance, the SOI nanopolarimeter may consist in a silicon waveguide designed to support two guided modes (TE-like and TM-like) at the operating wavelength and having a lateral protuberance on it (see Fig. 1b for the specific case of a T-block shaped scatterer). As discussed in the Supporting Information (Section S.3), breaking the mirror symmetry enables SOI and, thus, is essential for the device to perform as a nanopolarimeter. It can be shown that there exists an incident polarization $\mathbf{E}_{\max }^{k}$ for each port-mode combination $k$ that maximizes the corresponding effective area $A_{\mathrm{eff}}^{k}\left(\mathbf{E}_{\mathrm{max}}^{k}\right)=A_{\mathrm{max}}^{k}$ (Supporting Information, Section S.2). Fig. $1 \mathrm{~b}$ shows the four calculated polarization ellipses corresponding to $\mathbf{E}_{\max }^{T E+}, \mathbf{E}_{\max }^{T E-}, \mathbf{E}_{\max }^{T M+}$ and $\mathbf{E}_{\max }^{T M-}$, which maximize the effective area of the TE and TM-like modes propagating on the $+x$ or $-x$ direction on our designed structure, for the specific T-block scatterer at a wavelength $\lambda=1569 \mathrm{~nm}$. When the incident beam carries any one of these polarizations, the amplitude of one of the four components of the measured powers $\mathbf{P}$ will be maximum. Thus, $\mathbf{P}$ carries information about the relative amplitude of these four polarization components, from which the original SoP can be reconstructed as explained before. Explicitly, for a normalized incident polarization Jones vector $\mathbf{E}$, the power coupled into the $k$-th port-mode combination is equal to $P_{k}=I_{\mathrm{inc}} A_{k}^{\max }\left|\mathbf{E} \cdot\left(\mathbf{E}_{\max }^{k}\right)^{*}\right|^{2}$. This can be rewritten as a linear combination of the Stokes parameters of incident light $P_{k}=$ $\frac{1}{2} I_{\text {inc }} A_{k}^{\max } \mathbf{S}_{\text {max }}^{k} \cdot \mathbf{S}=\mathbf{a}_{k} \cdot \mathbf{S}$, where $\mathbf{a}_{k}$ will therefore be the $k$-th row of the polarimetric matrix $\mathbf{W}$ and $\mathbf{S}_{\max }^{k}$ corresponds to the Stokes parameters of the polarization $\mathbf{E}_{\max }^{k}$. This theoretical approach, useful for simulation and design, is derived in the Supporting Information. In the experiment, $\mathbf{W}$ is obtained through calibration as described below, and therefore will account for unequal waveguide losses and other experimental effects. 


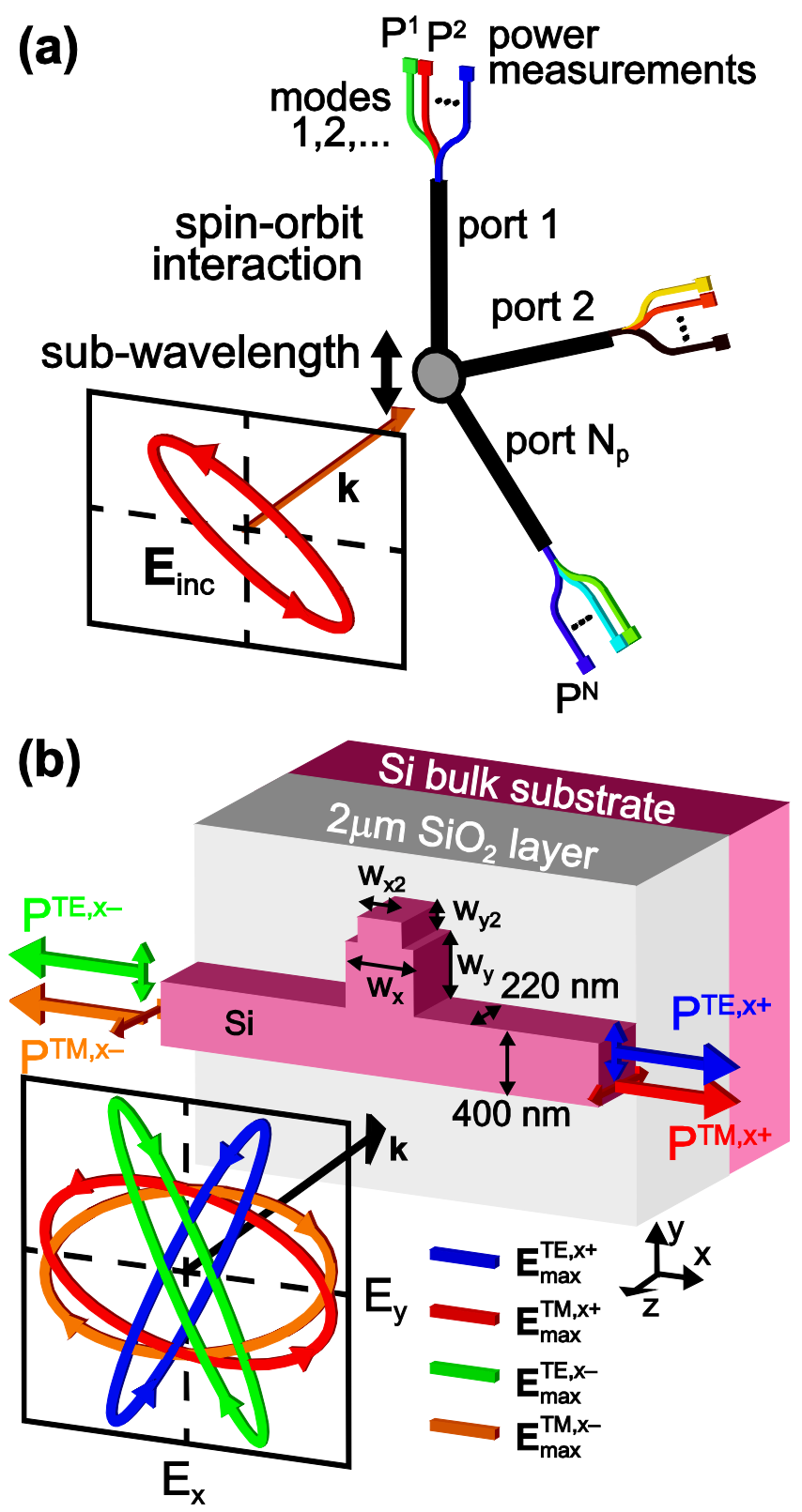

Figure 1. SOI-based Stokes nanopolarimetry. (a) A subwavelength scatterer illuminated by a transverse wave will scatter light into different modes of a set of waveguides. SOI allows the scattered power to depend on the input SoP, so measuring the output power for each mode will allow for complete reconstruction the input polarization. (b) In its simplest form, a SOI Stokes nanopolarimeter will consist on a bimodal silicon waveguide (supporting TE-like and TM-like guided modes) laterally perturbed by a scatterer, which breaks the mirror symmetry and enables SOI. The input polarizations providing maximum output power for the 4 different port-mode combinations at a wavelength $\lambda=1569 \mathrm{~nm}$ are depicted. This T-block shaped scatterer provides an optimal polarimetric response. 
If the scatterer-waveguide system is mirror symmetric with respect to the $x z$ and $y z$ planes, the matrix $\mathbf{W}$ becomes singular and the SoP cannot be retrieved. However, as long as the mirror symmetry is broken, SOI will make W invertible (see Supporting Information Section S.2 for details and a comparison between the symmetric and asymmetric case). However, bad conditioning of $\mathbf{W}$ may lead to inaccurate results mainly in the context of faint scattered signals that could be obscured by undesired noise, as it usually happens in nanoscale optical signals. Remarkably, the SOI nanopolarimeter can be easily designed to perform optimally, in the sense of minimizing the condition number of $\mathbf{W}$. For a polarimeter with four outputs with equal effective areas $A_{\max }^{k}$, this is equivalent to maximizing the volume of the tetrahedron inscribed in the Poincare sphere whose vertices are the four SoP's corresponding to the four different $\mathbf{E}_{\text {max }}^{i} \cdot{ }^{19,20}$ When the scatterer and the two output ports are mirror symmetric, as the " + " and "-" outputs in our structure and many previous SOI experiments, it follows that the effective areas of opposite outputs of the same mode are equal $A_{\text {eff }}^{+}\left(\mathbf{E}_{1}\right)=A_{\text {eff }}^{-}\left(\mathbf{E}_{2}\right)$ whenever $\mathbf{E}_{1}$ and $\mathbf{E}_{2}$ are related by the same mirror symmetry. We therefore need to design two polarizations $\mathbf{E}_{\max }^{T E+}$ and $\mathbf{E}_{\max }^{T M+}$ such that, together with their mirror symmetric counterparts $\mathbf{E}_{\max }^{T E-}$ and $\mathbf{E}_{\max }^{T M-}$ form an optimum set of polarizations that maximize the tetrahedron volume. The mathematical description of all optimal cases is provided in the Supporting information, Section S.2. In order to design an optimal nanopolarimeter, we performed numerical simulations starting with a rectangular scatterer whose polarization sorting was not optimal, as shown in Fig. 2a. ${ }^{12}$ We then applied a numerical optimization algorithm to finally get a T-block shaped scatterer having a set of polarization states (Fig. $2 b$ ) that define a tetrahedron with a volume that is $99.7 \%$ of the maximum attainable value, as shown in Fig. $2 \mathrm{c}$. 


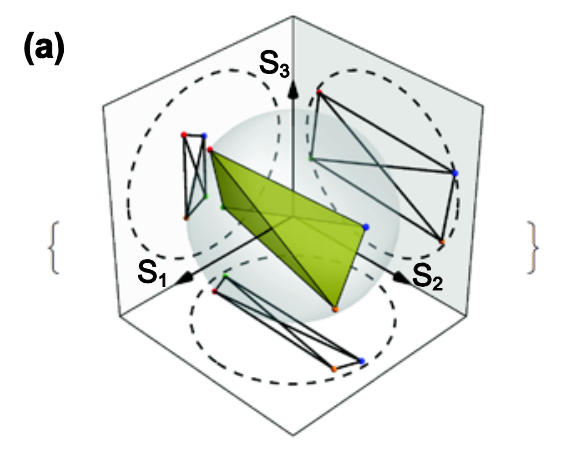

(b)
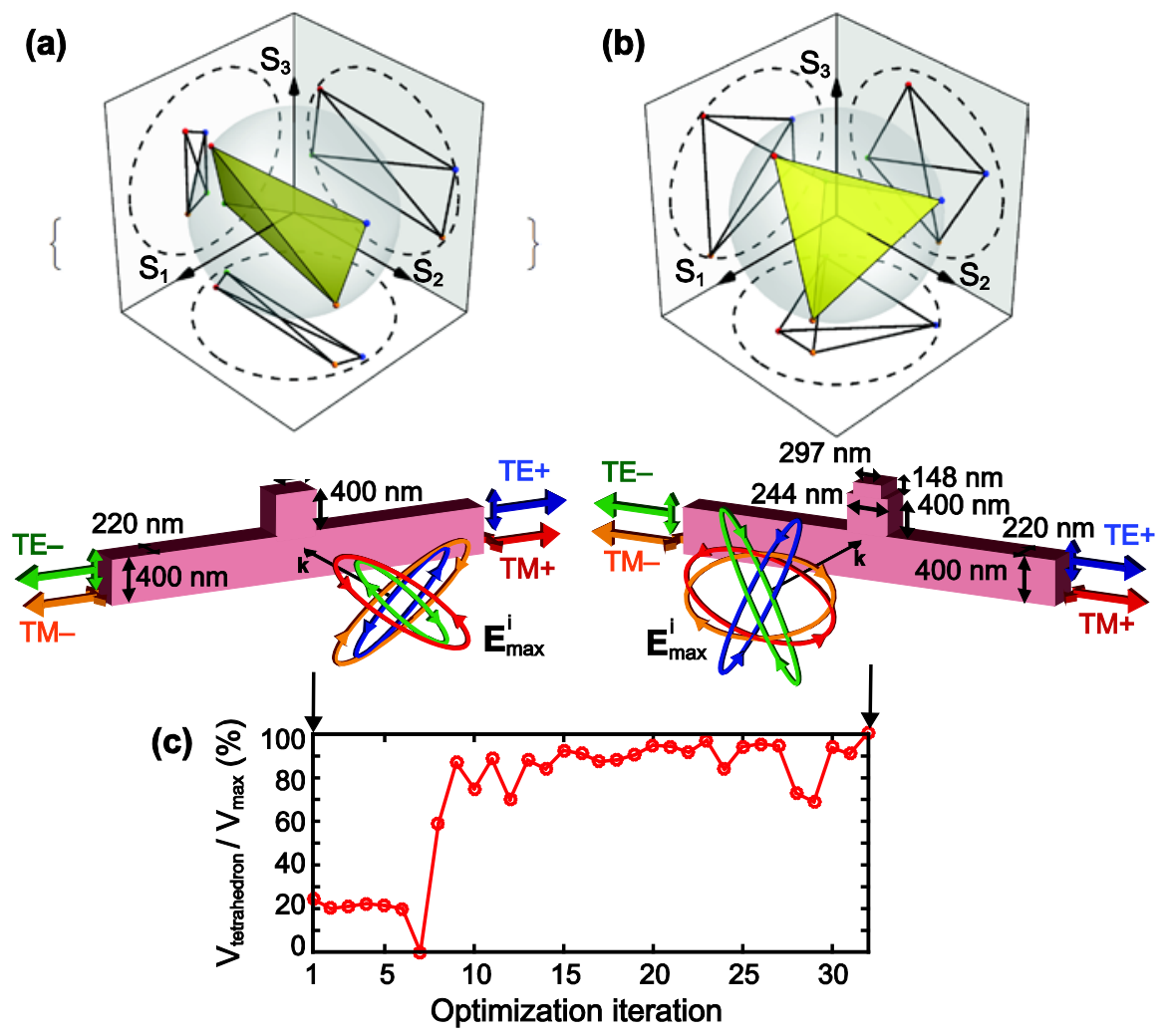

Figure 2. Optimal SOI nanopolarimeter. Starting from a rectangular scatterer asymmetrically coupled to the silicon waveguide in (a), we are able to find an optimal T-block scatterer shown in (b) via an optimization process that maximizes the volume of the tetrahedron inscribed within the Poincaré sphere. The optimization process, summarized in (c), is detailed in the Supporting information, Section S.3. The tetrahedrons for each scatterer-waveguide configuration as well as the polarizations giving rise to maximum optical power for the different port-mode pairs are depicted.

The optimal SOI nanopolarimeter was fabricated using standard Si-fabrication tools (Supporting information, Section S.4) and measured using the experimental set-up depicted in Fig. 3a. The sample was placed vertically, so that two orthogonal paths were created: the "vertical path" is the light scattered by our polarimeter into the integrated waveguides and towards the top edge of the sample, from which the device outputs could be measured using a microscope and a camera. The "horizontal path" corresponds to the illuminating light that propagates through the silicon substrate -transparent at the employed wavelengths- that we use to externally monitor the incident SoP with conventional optics. First the matrix $\mathbf{W}$ was formed from the calibration process (Supporting information, Section S.5) and then the active area of the nanopolarimeter was illuminated with a set of different input polarizations, where the angle, ellipticity and handedness were varied. All spot intensities $\mathbf{P}$ were captured for each input polarization and the SoP of the incident input light $\mathbf{S}$ was 
retrieved by applying $\mathbf{S}=\overline{\mathbf{W}}^{-1} \cdot \mathbf{P}$. These retrieved polarizations are shown in Fig. 3b. The agreement between the generated polarization (measured externally) and the polarization retrieved after measurement of the output power for each mode is remarkable, even for elliptical polarizations. Figure $3 c$ shows the matrix conditioning number $\kappa$ as a function of the wavelength obtained from simulations for both the initial and the optimized nanopolarimeters. It is seen that the optimal polarimeter reaches the minimum attainable condition number ${ }^{21} \kappa=\sqrt{N-1}=\sqrt{3}$ at $\lambda=1570 \mathrm{~nm}$ and performs quasi-optimally on a broad bandwidth ( $100 \mathrm{~nm})$. We also retrieved $\kappa$ from experiments for the optimal structure (see Fig. 3c). Although the obtained values (minimum 4) are not so small as for the numerical simulations (which we ascribe mainly to the fabricationinduced rounding of the scatterer, as seen in the SEM image, and to experimental imbalance between outputs, such as unequal propagation losses of the output waveguides), the general spectral shape shows a good qualitative correspondence with the calculated one. Notice that we were able to retrieve the SoP at other wavelengths, even though in this case the nanopolarimeter did not perform optimally (Supporting information, Section S.5). However, in general we observed a better accuracy for smaller values of $\kappa$.

Figure 4 shows the experimental performance of two fabricated and measured devices (the original rectangular-shaped polarimeter and the optimal T-block shaped polarimeter). Comparing the polarization ellipses of the recovered SoP (dashed line) with the input SoP measured manually from the experimental horizontal path (solid line), it can be seen that the accuracy is better for the optimal polarimeter, as expected from its careful design. 

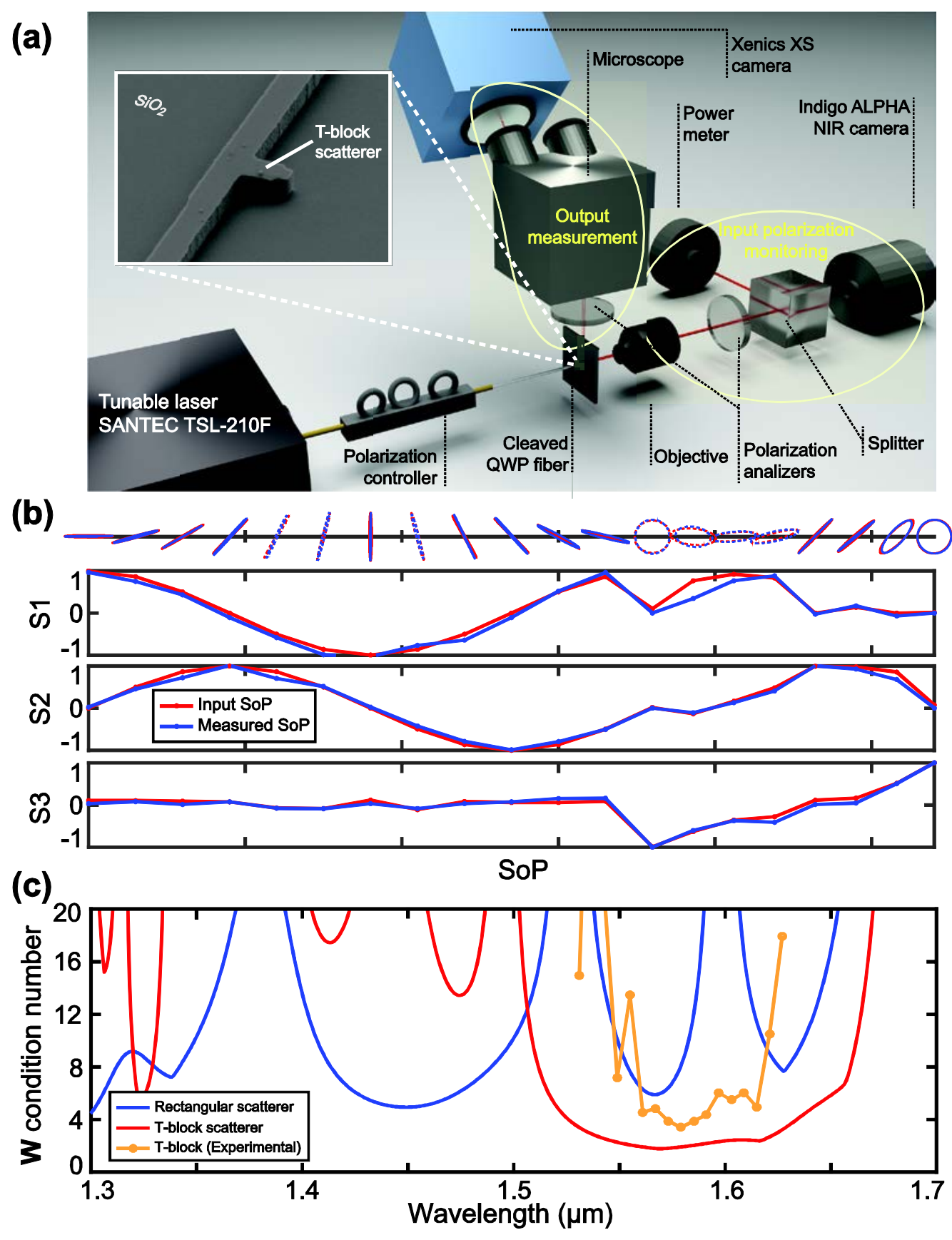

Figure 3. Experimental demonstration of the optimal nanopolarimeter with the T-block shaped scatterer. (a) Experimental setup (Inset: SEM image of one of the measured samples). (b) Retrieved polarization for a set of experiments performed at $\lambda=1558 \mathrm{~nm}$ : input (red) and retrieved (blue) SoP. (c) Condition number $\kappa$ of the matrix $\mathbf{W}$ as a function of the operation wavelength for the initial rectangular scatterer (simulation) and the optimal scatterer (simulation and experiment). 
(a)
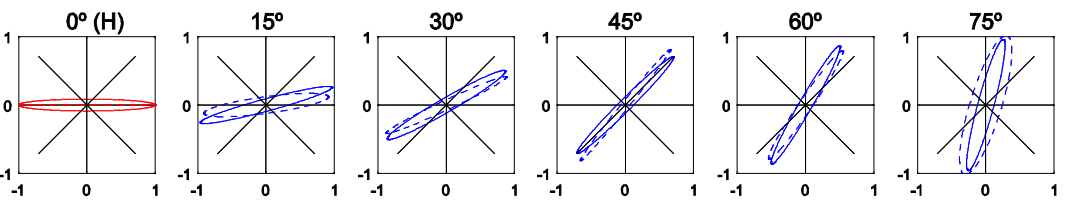

$400 \mathrm{~nm}$
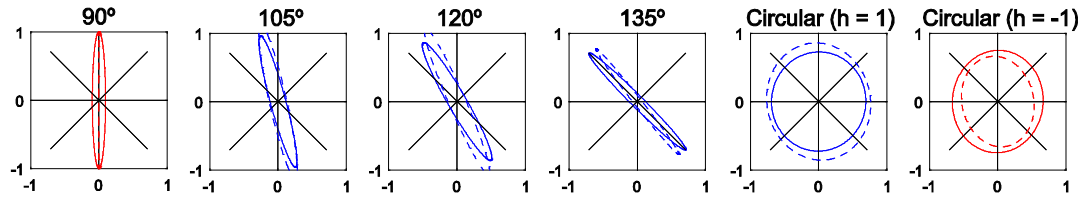

(b)
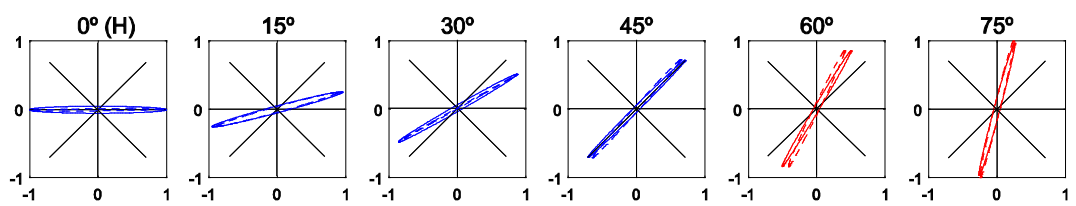

$354 \mathrm{~nm}$
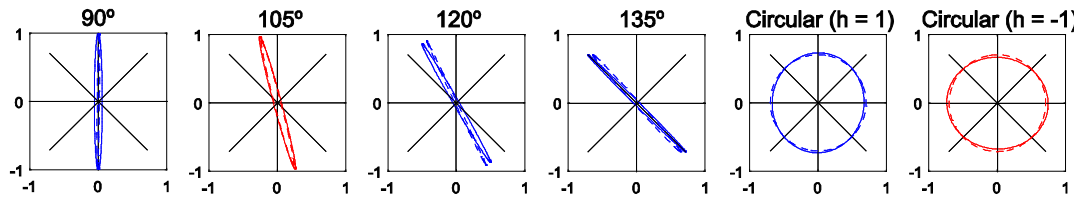

Figure 4. Measured polarization ellipses of the input (solid line) and recovered (dashed line) SoP for two different nanopolarimeters. (a) Response of the initial rectangular polarimeter at $\lambda=1550 \mathrm{~nm}$; (b) Response of the optimized T-block shaped polarimeter response at $\lambda=1558 \mathrm{~nm}$. Blue (red) lines represent left-handed (right-handed) polarization.

In the previous configuration, separation of the TE-like and TM-like guided modes is required to determine the Stokes parameters, which could be done on-chip via modal splitting architectures ${ }^{22}$ or by employing polarization filters after light has escaped from the chip (as in our experiments). In order to avoid conversion or filtering processes, other architectures following the general scheme of Fig. 1a could be implemented. For instance, a metallic scatterer asymmetrically deposited on top of a waveguide crossing (see Fig. 5a) could be employed. In this case, by measuring the total optical power (TM + TE modes) at each of the four output ports (see the SEM image of a fabricated sample in Fig. $5 \mathrm{~b}$ ) it is possible to retrieve the Stokes parameters of the incoming signal (Fig. 5c). Though the performance of the nanopolarimeter shown in Fig. 4 is not optimal, it could be optimized by using the same approach described above. 


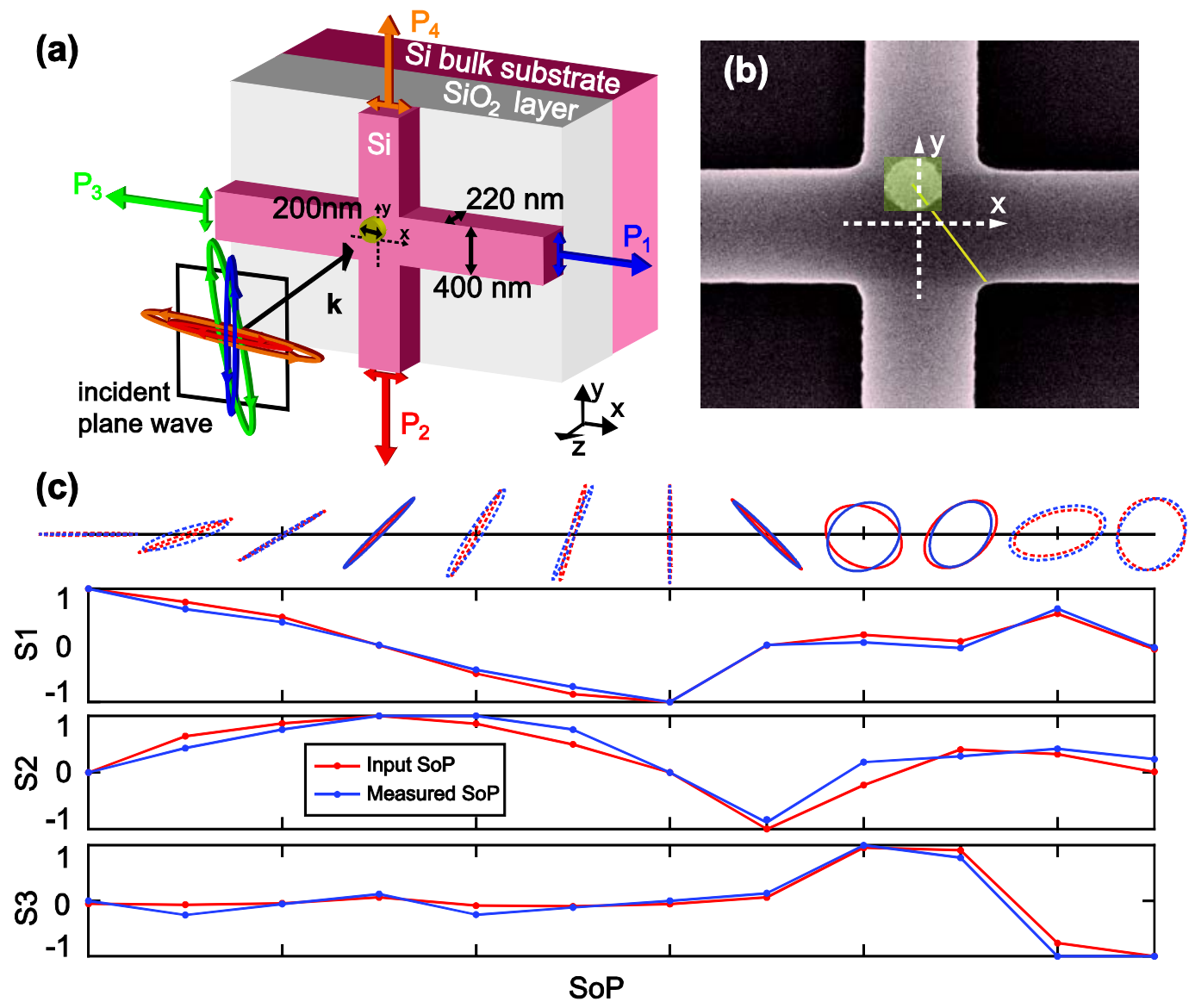

Figure 5. Cross-waveguide SOI nanopolarimeter. (a) Scheme of the nanopolarimeter consisting of a gold microdisk asymmetrically placed on top of a silicon waveguide crossing. The polarizations maximizing the outputs are also depicted (in this case, the nanopolarimeter does not perform optimally). (b) SEM image of a fabricated device (the gold microdisk has a diameter of $200 \mathrm{~nm}$ and a thickness of $30 \mathrm{~nm}$ ). (c) Experimental SoP retrieval at $\lambda=1550 \mathrm{~nm}$ : input (red) and retrieved (blue) SoP.

So far, we have focused on fully-polarized, transverse light beams. However, when monitoring processes at the nanoscale, we can usually find partly polarized light as well as beams having a nonnegligible longitudinal field component. Therefore, it makes sense to consider whether SOI polarimetry would work in such important cases. For the case of unpolarized light we should take into account that the lack of polarization will prevent the directional-scattering arising from SOI, so in the symmetric scatterer, light will be equally scattered along the $+x$ and $-x$ directions for each waveguide mode. As a result, we will retrieve $S_{1}=S_{2}=S_{3}=0$ and $S_{0}>0$, which means that SOI nanopolarimetry would also work for unpolarized or partially polarized light, as expected from any complete Stokes polarimeter. Concerning light with longitudinal field components, we notice that the guided modes have also components along such direction $\left(E_{2}\right)$ which enable the existence of SOI effects for this component. Therefore, although this kind of measurements falls beyond the Stokes 
parameters framework, it should also be possible to determine the amplitude and phase of $E_{\mathrm{z}}$ just by adding more paths (ports or modes) to the whole system.

Notice that polarimeters relying upon plasmonic nanoresonators ${ }^{23,24}$ and metasurfaces ${ }^{25-29}$ have been recently demonstrated. Despite their remarkable performance, they use metallic nanostructures with complex shapes, which besides introducing undesired losses, are not typically compatible with standard semiconductor fabrication technologies. More importantly, they do not allow local measurement of the SoP: whilst plasmonic resonators measure distinct polarization components in different places, metasurfaces are based on an extended, collective response of a set of scatterers. In contrast, our device, which is fully fabricated on a silicon chip without metals, makes use of the SOI taking place in a subwavelength scatterer meaning that SoP is detected locally. Moreover, our nanopolarimeter can be designed to operate optimally, which would reduce the effect of noise and random errors arising during the retrieval process. Notice that recently proposed metasurface-based polarimeters cannot operate optimally since the states they separate always describe a plane in the Poincaré sphere. ${ }^{26}$ Since the underlying silicon substrate is transparent at the operating wavelength, our device can be inserted directly on the optical path of a light beam, with only a negligible fraction of the incident power being sampled and used for measurement, while the rest of the beam propagates through the silicon chip. Such in-line operation with low insertion losses allows measurement of the SoP of a light beam in real time with little disturbance to the beam (nondestructive). This contrasts with recently proposed methods of local measurement of vector electric field that rely on collecting the scattered light and measuring it over a wide range of angles ${ }^{30}$ or using external polarization optics. ${ }^{31}$

A main feature of SOI nanopolarimetry is that the system outputs are integrated waveguides. Having the SoP information on waveguide modes offer a series of practical advantages: they potentially allow for on-chip measurement and SoP retrieval with no external optics or cameras; and the SoP mapping could be further optically processed in the waveguide. For instance, measuring the spectrum via on-chip spectrometers ${ }^{32}$ could lead to on-chip nano-spectropolarimetry. On the other hand, the use of waveguides imposes a limitation. Full local mapping of the SoP of inhomogeneous beams in real time via dense two-dimensional arrays becomes out of reach due to the existence of the waveguide. Still we could easily construct one-dimensional arrays with sub-micron spacing for real-time SoP scanning along a line. By moving the device with respect to the beam (using for instance a piezoelectric mount) two dimensional mapping of time-invariant beams becomes feasible. In summary, we have shown that SOI provides a general method to fully determine the polarization of a light beam locally, non-destructively, optimally and in a single shot. This approach shows a 
number of advantages such as optimal operation over a broad bandwidth, on-chip implementation in a single lithography step, and suitability for high-speed polarization measurement in an in-line configuration. The SOI Stokes nanopolarimetry method is universal: it can be applied to any frequency regime, being always subwavelength in size, and implemented in any technological platform. Together with the integrated approach for generation of arbitrary polarization states ${ }^{33}$, this nanopolarimeter completes a set of nanophotonic elements for full local polarization management at the nanoscale, unveiling the practical potential of the QSHE of light. ${ }^{10}$

\section{Associated content}

The theoretical description of the SOI Stokes nanopolarimetry concept together with fabrication and experimental methods as well as associated references are available in the Supporting Information.

\section{Acknowledgments}

We acknowledge support from the Spanish Ministry of Economy and Competiveness (MINECO) under grant TEC2014-51902-C2-1-R and the Valencian Conselleria d'Educación, Cultura i Esport under grant PROMETEOII/2014/034. F. J. R.-F. acknowledges support from the European Research Council under project ERC-2016-STG-714151-PSINFONI.

\section{References}

1. Bliokh, K. Y.; Rodríguez-Fortuño, F. J.; Nori, F.; Zayats, A. V. Nat. Photonics 2015, 9, 796-808.

2. Cardano, F.; Marrucci, L. Nat. Photonics 2015, 9, 776-778.

3. Aiello, A.; Banzer, P.; Neugebauer, M.; Leuchs, G. Nat. Photonics 2015, 9, 789-795.

4. Rodríguez-Fortuño, F. J.; Marino, G.; Ginzburg, P.; O’Connor, D.; Martínez, A.; Wurtz, G. A.; Zayats, A. V. Science 2013, 340, 328-331.

5. Lin, J.; Mueller, J. B.; Wang, Q.; Yuan, G.; Antoniou, N.; Yuan, X. C.; Capasso, F. Science 2013, 340, 331-334.

6. Petersen, J.; Volz, J.; Rauschenbeutel, A. Science 2014, 346, 67-71.

7. Rodríguez-Fortuño, F. J.; Barber-Sanz, I.; Puerto, D.; Griol, A.; Martínez, A. ACS Photon. 2014, 1, 762-767.

8. Söllner, I.; Mahmoodian, S.; Hansen, S. L.; Midolo, L.; Javadi, A.; Kiršanskè, G.; Pregnolato, T.; El-Ella, H.; Lee, E.H; Song, J. D.; Stobbe, S.; Lodahl, P. Nat. Nanotechnol. 2015, 10, 775778.

9. Bliokh, K. Y.; Bekshaev, A. Y.; Nori, F. Nat. Commun. 2014, 5, 3300.

10. Bliokh, K. Y.; Smirnova, D.; Nori, F. Science 2015, 348, 1448-1451.

11. Van Mechelen, T.; Jacob, Z. Optica 2016, 3, 118-126.

12. Rodríguez-Fortuño, F. J.; Puerto, D.; Griol, A.; Bellieres, L.; Martí, J.; Martínez, A. Opt. Lett. 2014, 39, 1394-1397.

13. Mitsch, R.; Sayrin, C.; Albrecht, B.; Schneeweiss, P.; Rauschenbeutel, A. Nat. Commun. 2014, $5,5713$.

14. Beckley, A. M.; Brown, T. G.; Alonso, M. A. Opt. Express 2010, 18, 10777-10785.

15. Bauer, T.; Banzer, P.; Karimi, E.; Orlov, S.; Rubano, A.; Marrucci, L.; Santamato, E; Boyd, R. W.; Leuchs, G. Science 2015, 347, 964-966. 
16. Femius Koenderink, A.; Alù, A.; Polman, A. Science 2015, 348, 516-521.

17. Espinosa-Soria, A.; Martinez, A. IEEE Photon. Technol. Lett. 2016, 28, 1561-1564.

18. Chipman, R. A. "Polarimetry," in Handbook of Optics, M. Bass, ed. The McGraw-Hill Companies, Inc., 2010.

19. Azzam, R. M. A.; Elminyawi, I. M.; El-Saba, A. M. J. Opt. Soc. Am. A 1988, 5, 681-689.

20. Foreman, M. R.; Favaro, A.; Aiello, A. Phys. Rev. Lett. 2015, 115, 263901.

21. Tyo, J. S. Appl. Opt. 2002, 41, 619-630.

22. Fukuda, H.; Yamada, K.; Tsuchizawa, T.; Watanabe, T.; Shinojima, H.; Itabashi, S. I. Opt. Express 2006, 14, 12401-12408.

23. Afshinmanesh, F.; White, J. S.; Cai, W.; Brongersma, M. L. Nanophotonics 2012, 1, 125-129.

24. Xie, Y.-B.; Liu, Z.-Y.; Wang, Q.-J.; Zhu, Y.-Y.; Zhang, X.-J. Appl. Phys. Lett. 2014, 105, 101107.

25. Wen, D.; Yue, F.; Kumar, S.; Ma, Y.; Chen, M.; Ren, X.; Kremer, P. E.; Gerardor, B. D.; Taghizadeh, M. R.; Buller, G. S.; Chen, X. Opt. Express 2015, 23, 10272-10281.

26. Balthasar Mueller, J. P.; Leosson, K.; Capasso, F. Optica 2016, 3, 42-47.

27. Pors, A.; Nielsen, M. G.; Bozhevolnyi, S. I. Optica 2015, 2, 716-723.

28. Lepetit, T.; Kanté, B. Nature Photon. 2015, 9, 709-710.

29. Maguid, E.; Yulevich, I.; Veksler, D.; Kleiner, V.; Brongersma, M. L.; Hasman, E. Science 2016, 352, 1202-1206.

30. Bauer, T.; Orlov, S.; Peschel, U.; Banzer, P.; Leuchs, G. Nat. Photonics 2014, 8, 23-27.

31. Rodríguez-Herrera, O. G.; Lara, D.; Bliokh, K. Y.; Ostrovskaya, E. A.; Dainty, C. Phys. Rev. Lett. 2010, 104, 253601.

32. Redding, B.; Liew, S. F.; Sarma, R.; Cao, H. Nat. Photonics 2013, 7, 746-751.

33. Rodríguez-Fortuño, F. J.; Puerto, D.; Griol, A.; Bellieres, L.; Martí, J.; Martínez, A. Laser Photonics Rev. 2014, 8, L27-L31.

TOC Figure

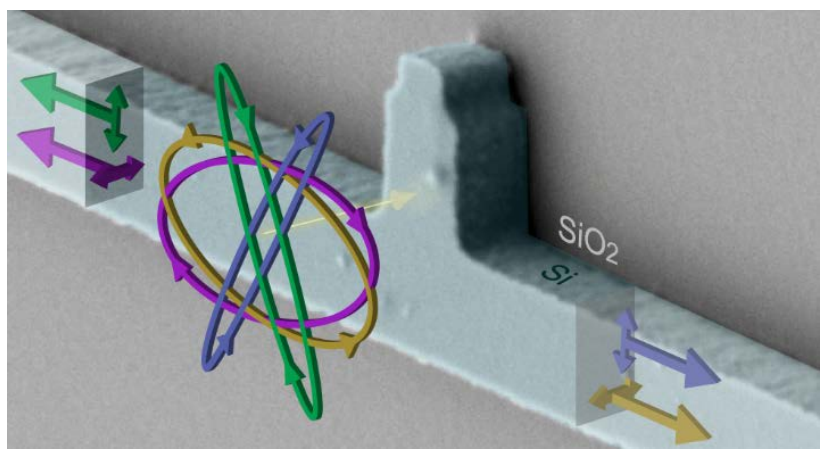

